\title{
Can Dexmedetomidine prevent delirium during the weaning process in critically ill patients?
}

Fotopoulou G., Poularas I., Alexopoulou A., Kalogeromitros A., Papakonstantinou K., Koukoulitsios G. General Hospital of Athens - G. Gennimatas, Dept of Intensive Care, Athens, Greece

\section{Background and Goal of study}

Intensive Care Unit (ICU) delirium is associated with longer length of ICU stay and higher incidence of mortality (1). Dexmedetomidine (DEX) is an alpha-2-adrenergic agonist recently introduced as a sedation adjunct. The objective of our study was to evaluate the efficacy of DEX in preventing delirium, either hyperactive or hypoactive, during the weaning process.

\section{Materials and Methods}

We performed a randomized controlled study in our multidisciplinary ICU. Adult patients mechanically ventilated, meeting the standard criteria for weaning were enrolled and randomized to receive either DEX $(0.15-1.5 \mathrm{mcg} / \mathrm{kg} / \mathrm{h})$ or remifentanil $(0.5-15 \mathrm{mcg} / \mathrm{kg} / \mathrm{h})$ in order to achieve a Richmond Agitation-Sedation Scale of -2 to 0 . Patients with a history of alcohol, opioid or benzodiazepine abuse were excluded. Illness severity scoring systems (APACHE II and SOFA) were calculated on the first ICU day. Delirium was assessed daily according to the Confusion Assessment Method for the ICU. Time to extubation and vital parameters were also recorded. Continuous variables were compared using the Student t-test. Categorical variables were compared using the chi-square test or Fisher's exact test.

\section{Results and Discussion}

Fifty-two mechanically ventilated, critically ill patients were included in the study (median age 69 years, 55\% were males). Median values for APACHE II and SOFA scores were 18 and 9, respectively. The use of DEX was associated with significantly better extubation quality compared to remifentanil reflected in the prevalence of delirium during the weaning process [23\% $(6 / 26)$ vs $65 \%(17 / 26), p<0.01$ ], as well as after extubation [ $8 \%(2 / 26)$ vs $35 \%(9 / 26), p<0.01$ ]. Consequently, less additional rescue medications for agitation were applied in the DEX group [19\% $(5 / 26)$ vs $58 \%(15 / 26)]$. Significant differences between the two groups were recorded in the median time to extubation ( $40.8 \pm 36$ hours in the DEX group vs $60.0 \pm 28.8$ hours in the remifentanil group, $p<0.05)$ and ICU length of stay $(19.9 \pm 8.45$ days in the DEX group vs $15.2 \pm 11.7$ days in the remifentanil group, $p<0.05$ ). However, the hemodynamic adverse effects (hypotension, hypertension, bradycardia) were reported to be less common in the remifentanil group [ $42 \%$ $(11 / 26)$ vs $35 \%(9 / 26), p=0.175)$, although not significantly.

Dexmedetomidine seems to have clinical benefits in facilitating weaning process due to the lower incidence of delirium with minimal adverse events.

\section{Conclusions}

1. Ely EW et al. JAMA 2004; 291:1753-1762. 